\title{
Periodic Body-and-Bar Frameworks
}

\section{$\operatorname{AUTHOR}(S)$ :}

Borcea, Ciprian; Streinu, Ileana; Tanigawa, Shinichi

\section{CITATION:}

Borcea, Ciprian ... [et al]. Periodic Body-and-Bar Frameworks. SIAM Journal on Discrete Mathematics 2015, 29(1): 93-112

ISSUE DATE:

2015-01-15

URL:

http://hdl.handle.net/2433/200696

RIGHT:

(C) 2015, Society for Industrial and Applied Mathematics 


\title{
PERIODIC BODY-AND-BAR FRAMEWORKS*
}

\author{
CIPRIAN BORCEA ${ }^{\dagger}$, ILEANA STREINU ${ }^{\ddagger}$, AND SHIN-ICHI TANIGAWA ${ }^{\S}$
}

\begin{abstract}
Periodic body-and-bar frameworks are abstractions of crystalline structures made of rigid bodies connected by fixed-length bars and subject to the action of a lattice of translations. We give a Maxwell-Laman characterization for minimally rigid periodic body-and-bar frameworks in terms of their quotient graphs. As a consequence we obtain efficient polynomial time algorithms for their recognition based on matroid partition and pebble games.
\end{abstract}

Key words. periodic frameworks, rigidity, matroids, crystalline structure

AMS subject classifications. 52C25, 05B35, 74E15

DOI. $10.1137 / 120900265$

1. Introduction. In this paper, we study $d$-dimensional periodic frameworks made of rigid bodies connected with rigid bars. Figure 1 gives an example. We prove a combinatorial characterization for the quotient graphs of minimally rigid frameworks, in terms of matroid unions of graphs satisfying Maxwell-sparsity conditions. This leads to efficient, polynomial time algorithms for their recognition, based on matroid partition and pebble games.

Periodic structures are naturally associated with crystallographic studies [23]. The central role played by a periodicity lattice and corresponding fundamental domains was recognized even before X-ray diffraction opened up experimental possibilities. Crystals known as framework materials $[26,9,27]$ have distinctive substructures which can be modeled and investigated as articulated systems made of rigid pieces and joints described abstractly by an infinite periodic graph. Similar models appear in structural engineering in connection with infinite trusses, foams, or cellular materials $[6,13,8$, 16]. While a fair number of traditional areas of mathematics are related or dedicated to lattice structures and periodicity, from crystallographic groups and quadratic forms to the geometry of numbers and sphere packings [15, 38,4], mathematical studies of periodic frameworks are relatively recent $[20,5,1]$.

Under variations of temperature or pressure, crystalline materials may undergo structural changes while still retaining periodicity. Understanding these phase transitions remains a challenging problem [7]. The role of geometry is particularly relevant in displacive phase transitions, which may be interpreted as continuous deformations of a given framework [9]. A rigorous mathematical understanding of rigidity and flexibility properties of periodic frameworks may assist, clarify, and complement approaches based on computational physics and materials science $[18,1,3]$.

\footnotetext{
${ }^{*}$ Received by the editors November 26, 2012; accepted for publication (in revised form) September 10, 2014; published electronically January 15, 2015. A preliminary version of this paper appeared in Proceedings of the 28th Annual ACM Symposium on Computational Geometry (SoCG), Chapel Hill, NC, 2012, pp. 347-356. The research of the first two authors was supported by a DARPA "23 Mathematical Challenges" grant, under the topic "Algorithmic Origami and Biology," and by NSF CCF-1319366.

http://www.siam.org/journals/sidma/29-1/90026.html

$\dagger$ Department of Mathematics, Rider University, Lawrenceville, NJ 08648 (borcea@rider.edu).

${ }_{\ddagger}^{\ddagger}$ Department of Computer Science, Smith College, Northampton, MA 01063 (istreinu@smith.edu). The research of this author was supported by NSF grant CCF-1016988.

$\S$ Research Institute for Mathematical Sciences, Kyoto University, Kyoto 606-8501, Japan (tanigawa@kurims.kyoto-u.ac.jp). The research of this author was supported by Grant-in-Aid for JSPS Research Fellowships for Young Scientists and by Kyoto University Start-Up Grant for Young Researchers.
} 


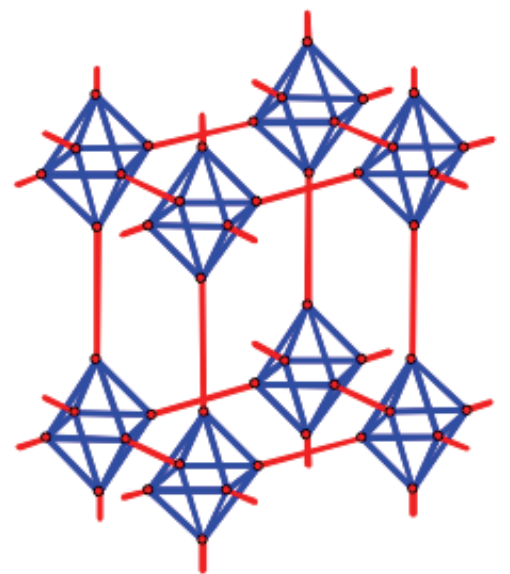

FIG. 1. Fragment of a periodic body-and-bar framework associated to Prussian blue crystalline materials [12]. Bodies are shown as octahedra. The framework is flexible and its geometric deformations are determined by the system of quadratic equations (2.1) described below. A bar-and-joint model and the deformation theory of [1] may also be used for this purpose.

Rigidity and flexibility studies of macromolecules modeled as mechanical (finite or periodic) frameworks $[12,18]$ are often conducted with computationally expensive yet numerically imprecise simulations. For finite structures characterized by theorems of Maxwell-Laman type (described below), much faster approaches based on degreeof-freedom counting and rigid component calculations are known. But such theorems are rare and difficult to obtain. For periodic structures, an appropriate mathematical formulation was presented only recently [1]. It opened the way to a combinatorial treatment and efficient algorithms [2] for periodic bar-and-joint structures (made from rigid bars connected through rotatable joints) in arbitrary dimension $d$.

The approach adopted in this paper is rooted in the theory developed in these recent papers [1,2], where a Maxwell-Laman theorem for periodic bar-and-joint rigidity was given. This approach reduces both the long-standing finite case and several other periodic situations to genericity refinement conjectures. It may be useful, in this context, to give an overview of some specific aspects and challenges related to periodic frameworks.

Modeling crystalline frameworks. Because of interatomic bond length and angle constraints, a crystalline material such as the one illustrated in Figure 1 can be treated as a periodic framework made from rigid bars connected at vertices, to which, in principle, the theory developed in $[1,2]$ applies. Often, substructures making individual rigid bodies are identified from the outset, such as oxygen tetrahedra in zeolites, the octahedra in Figure 1, or, in a 2D example, the gray bodies from Figure 2(b). In this paper we focus on structures made of rigid bodies connected by rigid bars.

Maxwell-Laman sparsity conditions. The theory of finite frameworks has a distinguished tradition going back almost 150 years to Maxwell [25], where a sparsity condition was shown to be necessary for minimal rigidity of bar-and-joint frameworks: in dimension $d$, for any subset of $d \leq n^{\prime} \leq|V|$ vertices, the graph should span at most $d n^{\prime}-\left(\begin{array}{c}d+1 \\ 2\end{array}\right)$ edges, with equality for the whole set of $n=|V|$ vertices. Its sufficiency for generic frameworks in dimension 2 was proven more than 100 years later (Laman's theorem [21]) and is known to fail in higher dimensions. The problem of completing the combinatorial characterization for bar-and-joint frame- 

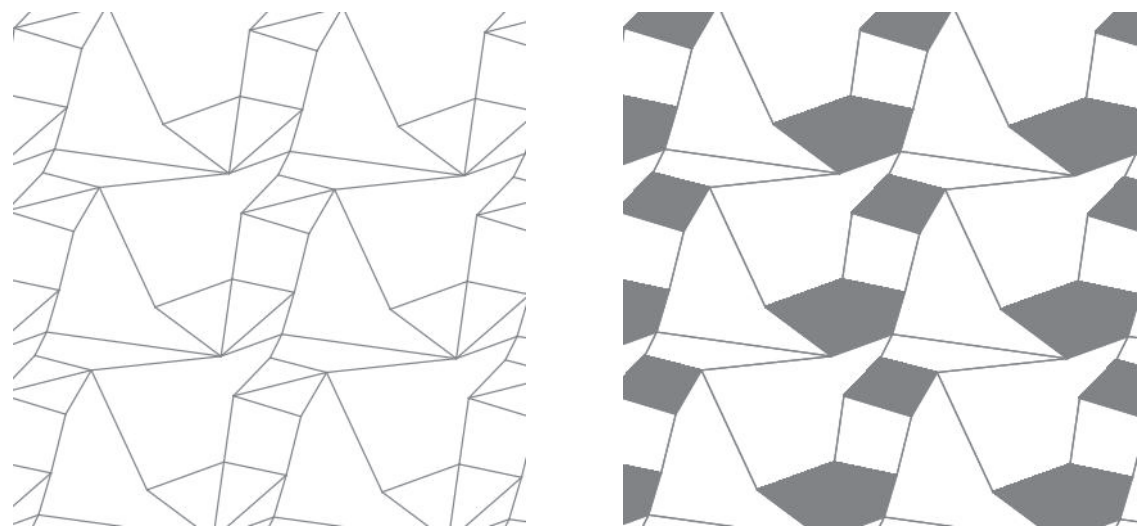

FIG. 2. Left: A $2 D$ periodic bar-and-joint framework containing smaller rigid components. Right: The same framework, viewed as a body-and-bar framework; the rigid components form the bodies, and the remaining bars connect distinct bodies.

works in arbitrary dimensions remains the most conspicuous open question in rigidity theory. However, some restricted classes of finite frameworks have been shown to have similar Maxwell-Laman counting characterizations: body-and-bar and bodyand-hinge frameworks [17, 39, 35, 34, 32], panel-and-hinge frameworks [19], and ( $d-2)$ dimensional plate-and-bar frameworks [36, 35, 32].

Generic structures. All known theorems giving combinatorial characterizations of framework rigidity proceed by associating a rigidity matrix $M$ to the framework. A set of algebraic constraints (e.g., fixed distances between some pairs of points) are applied on a collection of rigid objects (e.g., a finite set of points), with the goal of obtaining a rigid structure, i.e., one in which all the pairwise distances are determined. Linearizing on the tangent space at a specific point of the variety defined by these algebraic constraints leads to a matrix, called the rigidity matrix, whose rank we seek to determine. The maximal number of independent constraints that can be imposed is also the minimum number that would make the structure rigid. Together with additional constraints that eliminate the trivial rigid motions, they lead to a square rigidity matrix. Maximal rank, attained when the determinant $\operatorname{det}(M)$ of the rigidity matrix is nonzero, indicates that the framework is minimally rigid: it is rigid and the removal of any constraint will make it flexible. The condition of maximal rank is therefore an algebraic condition in the free variables of the system.

In algebraic geometry, a property which holds on the complement of some proper algebraic subvariety of an irreducible variety is called generic. Thus, if a property expressed by algebraic conditions holds at one point of an irreducible parameter variety, it becomes a generic property. In our context, the parameter spaces given by the free variables of the systems under consideration are typically products of affine spaces, and hence are irreducible, and maximal rank at one point implies maximal rank generically. With the term generic understood in this classical sense, we see that minimal rigidity is a generic property and thus inherently a graph-determined property. As soon as we have found one minimally rigid framework, all generic frameworks with the same graph will be minimally rigid.

This discussion extends naturally to frameworks whose rigidity matrix is not square, by expressing the rank condition in terms of corresponding minors of $M$. Some situations, as already addressed in [2], involve generic liftings of edges from a quotient 
graph to a periodic graph covering. At first sight, the parameters implicated here belong to some lattice $Z^{n}$, rather than some affine space. However, given the fact that a polynomial vanishing on $Z^{n} \subset R^{n}$ must vanish on $R^{n}$, the notion of genericity is not affected by the requirement of integer coordinates, rather than arbitrary coordinates.

Theorems of Maxwell-Laman type for minimally rigid structures characterize classes of graphs underlying frameworks whose rigidity matrices have maximum rank, generically, for certain structural models as described above. A random geometric realization of a graph belonging to such a class is, with probability 1, rigid. But the measure-zero set, when it is not rigid, is in general nonempty and may contain important cases that appear in practice. Refining the genericity analysis in order to prove that certain types of configurations remain rigid is in general a very difficult problem. An important result of this nature is the recent proof of the molecular conjecture [19]. Our contribution here, as a refinement of genericity conditions, is of a similar flavor.

The connection pattern of a periodic body-and-bar system determines an infinite multigraph $G=(V, E)$, with vertices corresponding to bodies and edges corresponding to bars between pairs of connected bodies. See Figure 2 for an example in two dimensions and Figure 1 for one in three dimensions. Periodicity or, more precisely, $d$ periodicity (where $d$ represents the dimension of the ambient space in which the graph is realized geometrically), requires a free Abelian automorphism subgroup $\Gamma \subset A u t(G)$ of rank $d$. We work under the assumption that the quotient graph $G / \Gamma$ has a finite number $n$ of vertex orbits and a finite number $m$ of edge orbits.

Rigidity for periodic frameworks, when addressed at the quotient level, is notably different from the finite case: The quotient graphs obtained from minimally rigid periodic bar-and-joint frameworks have been characterized in all dimensions by a Maxwell-type sparsity condition [2]. Although finite bar-and-joint frameworks, as well as periodic body-and-bar or body-and-hinge structures, can be reinterpreted as periodic bar-and-joint frameworks, they constitute special classes where additional algebraic dependencies are present. As indicated, such cases are not covered by the genericity assumptions of the periodic bar-and-joint result. Even obtaining a necessary sparsity condition may be nontrivial.

An additional difficulty, relevant from an algorithmic point of view, arises from the fact that not all types of sparsity conditions are matroidal. For instance, Maxwellsparsity for finite 3D bar-and-joint frameworks (the "3n-6" condition) is not. However, for the periodic body-and-bar structures studied in this paper, the conditions are matroidal. In this case, efficient recognition algorithms exist. Relevant for our setting are matroid partition [10] and pebble game algorithms [22].

Main result. We prove in section 4 the following result of Maxwell-Laman type.

TheOREM 1.1. A multigraph $G$ with $n$ vertices and $m=\left(\begin{array}{c}d+1 \\ 2\end{array}\right)(n-1)+d^{2}$ edges is the quotient graph of an infinitesimally rigid periodic body-and-bar framework in $\mathbb{R}^{d}$ under the action of a periodicity group $\Gamma$ if and only if it satisfies one, and hence both, of the following equivalent conditions:

(i) it decomposes into a disjoint union of two graphs on the full set of $n$ vertices, one with $d n-d$ edges and sparsity type and the other with $\left(\begin{array}{c}d \\ 2\end{array}\right) n+\left(\begin{array}{c}d+1 \\ 2\end{array}\right)$ edges and sparsity type;

(ii) it contains the union of two edge-disjoint graphs on the full set of $n$ vertices, one with $d n-d$ edges and sparsity type, and the other with $\left(\begin{array}{l}d \\ 2\end{array}\right) n$ edges and sparsity type.

In the rest of the paper, we formally define the periodicity model, identify the 
necessary sparsity conditions, prove their sufficiency, and, finally, give efficient algorithms for deciding rigidity, based on matroid partition and pebble games. As already seen in the bar-and-joint case [1,2], the mutual relations between the finite and the periodic settings are intricate. We emphasize here that our approach considers the periodicity group $\Gamma$ as part of the initial data $(G, \Gamma)$ but allows the variation of its representation as a lattice of translations of full rank. Moreover, the problem of minimal rigidity may be formulated at several levels. The most basic level looks only at the structure of the quotient graph $G / \Gamma$ and provides characterizations "up to a generic lifting of edges" from $G / \Gamma$ to a covering periodic graph. The main results of this paper are formulated at this level. For $n=1$, that is, in the case of a single body orbit under $\Gamma$, an answer is given at all levels and offers a first measure of the contrast between solving in terms of $G / \Gamma$ or fully in terms of the given periodic graph $(G, \Gamma)$. We discuss aspects implicated at this higher level after solving the problem at the basic level.

We further generalize Theorem 1.1, in arbitrary dimension $d$, by considering not just $d$-dimensional bodies but also a mixture of lower-dimensional "plates." The precise definitions and the statement of the theorem will be given in section 5 .

Related work. Periodic rigidity has been considered recently by other authors, in settings that differ from ours in several aspects. When the periodicity lattice is considered fixed, periodic frameworks are equivalent with frameworks on a fixed flat torus, and this is the viewpoint adopted in [30]. This thesis deals primarily with bar-and-joint frameworks in dimension 2 , but in its final part it also has some considerations about body-and-bar frameworks on such a "fixed torus." A necessary condition for minimal rigidity is presented and conjectured to be also sufficient. Recently, solutions to the conjecture appeared in [31] for the 3D case and in [33] for arbitrary dimension.

The deformable lattice formulation of [1] was adopted in [24], where periodic bar-and-joint frameworks are studied in the special case of dimension 2. A characterization of minimal rigidity is obtained in terms of the full periodic graph $(G, \Gamma)$. This result relies on the fact that finite rigidity has a combinatorial characterization in $2 \mathrm{D}$ (Laman's theorem), and, at this time, does not appear to be generalizable to higher dimensions.

Overview of the paper. We describe our setting in section 2, where we also derive the rigidity matrix. We indicate different levels for the problem of minimal rigidity in section 3 and solve it at all levels for the base case $n=1$. The main result characterizing minimal rigidity in terms of the structure of the quotient graph $G / \Gamma$ is proven in section 4 . In section 5 , we generalize our main theorem to mixed periodic plate-and-bar frameworks. In section 6 we discuss minimal rigidity in terms of the full periodic graph $(G, \Gamma)$ and formulate an open problem. Algorithmic consequences are presented in section 7 . In the concluding section 8 we mention a few other related open problems.

2. Periodic frameworks. In this section we introduce our formal definition of periodic rigidity for periodic body-and-bar frameworks. For standard rigidity theoretic definitions such as infinitesimal rigidity and rigidity matrix, see $[1,39]$.

We remind the reader of a classical concept from finite rigidity theory: A finite body-and-bar $d$-framework is a finite collection of rigid bodies in $\mathbb{R}^{d}$ connected through bars. The endpoints of a bar lie on two distinct bodies and act as rotatable joints, i.e., they permit rigid motions of the incident objects (bodies or bars) relative to each other, while contact is maintained at the joints. Several bars may be placed 

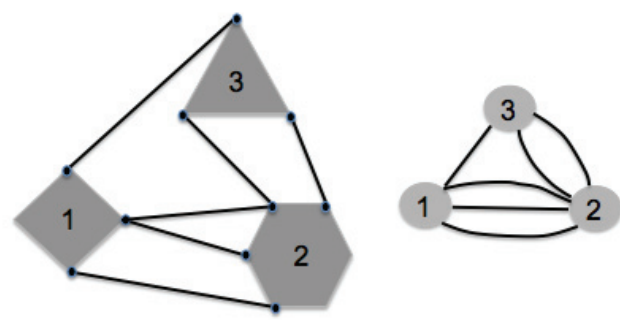

FIG. 3. A finite, minimally rigid body-and-bar framework in two dimensions, with three bodies and six bars, and its associated multigraph.

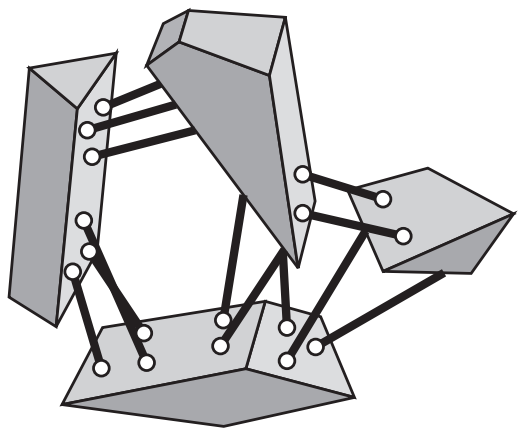

FIG. 4. A finite body-and-bar framework in three dimensions.

between the same pair of bodies. See Figures 3 and 4 . The combinatorial (incidence) structure of such a framework is captured by a multigraph $G=(V, E)$, whose vertices correspond to bodies and edges to bars.

Body-and-bar frameworks have been studied in several foundational papers (e.g., $[34,35])$ and have practical application in studies of molecular flexibility [37, 11]. We proceed now to our new definitions.

A $d$-periodic body-and-bar graph is a pair $(G, \Gamma)$ of an infinite multigraph $G=$ $(V, E)$ and a group $\Gamma$ acting on it. The group $\Gamma$, called the periodicity group of $G$, is a rank $d$ free Abelian subgroup of the automorphism group $A u t(G)$ of $G$, which acts without fixed points and has a finite number of vertex and edge orbits. The elements $\gamma \in \Gamma$ may be called periods of $G$. See Figure 5 for an example. Since both $n=\operatorname{card}(V / \Gamma)$ and $m=\operatorname{card}(E / \Gamma)$ are finite, this setting induces a finite quotient multigraph $G / \Gamma=(V / \Gamma, E / \Gamma)$.

Isometries. We denote by $E(d)$ the Euclidean group in dimension $d$, that is, the isometry group of the Euclidean space $\mathbb{R}^{d}$. The connected component of the identity, denoted by $S E(d)$, is made of all the orientation preserving isometries and is referred to as the special Euclidean group or the group of rigid motions in $\mathbb{R}^{d}$. The subgroup of translations is denoted by $\mathcal{T}\left(\mathbb{R}^{d}\right) \subset S E(d)$. A transformation in $E(d)$, respectively, $S E(d)$, can be represented by a pair $T=(p, M)$, where $p \in \mathbb{R}^{d}$ denotes a translation and $M \in O(d)$, respectively, $M \in S O(d)$, is an orthogonal transformation, respectivey, a special orthogonal one. $M$ is an orthogonal matrix with $|\operatorname{det}(M)|=1$, respectively, $\operatorname{det}(M)=1$. A Euclidean transformation $T: \mathbb{R}^{d} \rightarrow \mathbb{R}^{d}$ operates by the formula $T(x)=M x+p$. In this notation, translations correspond to pairs $\left(p, I_{d}\right)$, where $I_{d}$ is the $d \times d$ identity matrix.

To define a body-and-bar framework, we specify a placement in $\mathbb{R}^{d}$ for the bodies. 


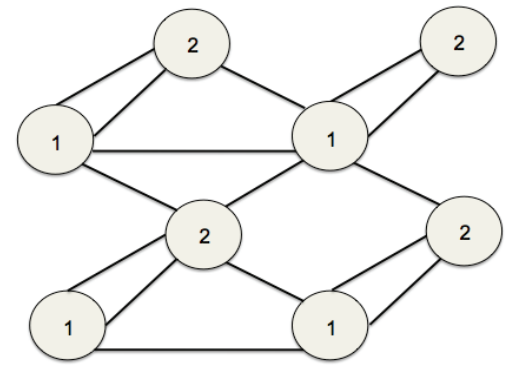

FIG. 5. A small fragment of an abstract periodic multigraph. Only the edges between depicted vertices are drawn. Equivalent vertices under the action of the group of periods have the same label. A realization is shown in Figure 6 and the quotient multigraph in Figure 7.
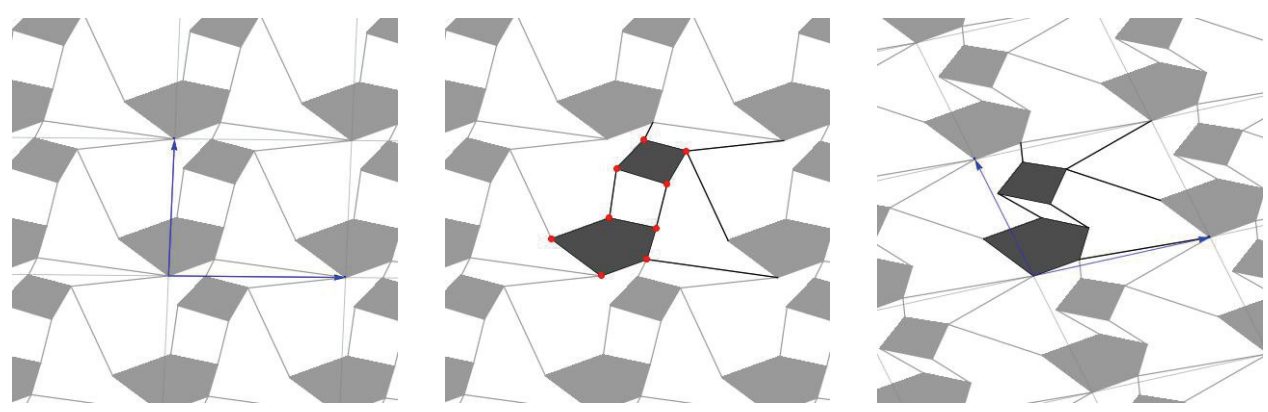

Fig. 6. Left: Fragment of a 2-periodic body-and-bar framework $(d=2)$, with the generators (which are not bars) of the periodicity lattice marked by arrows and the orbit of one bar endpoint visualized as a "lattice." Center: Representatives of the $n=2$ equivalence classes of bodies and $m=6$ classes of bars are shown in darker shades. Right: The framework is periodically flexible, as illustrated by this deformation, affecting both the framework and the periodicity lattice.

Each such placement is described by a pair $T=(p, M) \in S E(d)$ of a translation vector $p$ and an orthogonal matrix $M$. In other words, we conceive of the body as described by a (positively oriented) Cartesian frame with origin at $p$ and basis vectors corresponding to the columns of the orthogonal matrix $M \in S O(d)$.

Our next goal is to define a periodic body-and-bar framework. A 2D example is given in Figure 6.

A presentation in $\mathbb{R}^{d}$ of a $d$-periodic body-and-bar graph $(G, \Gamma)$ is given by an assignment of Cartesian frames to the vertices $\tau: V \rightarrow S E(d)$, together with an injective representation of the periodicity group $\Gamma, \pi: \Gamma \rightarrow \mathcal{T}\left(\mathbb{R}^{d}\right)$. We refer to $\pi(\Gamma)$ as the lattice of periods and require that it have rank $d$. Furthermore, for each edge $e=(i, j) \in E$, two (arbitrary but fixed) endpoints are given, indicating where the bar is attached on the bodies corresponding to vertices $i$ and $j$.

Let us denote these endpoints with $q^{i}=q^{i}(e), q^{j}=q^{j}(e)$. The coordinates $q^{i} \in \mathbb{R}^{d}$ are given with respect to the Cartesian frame marking the body corresponding to $i$, so that for a frame $\tau(i)=\left(p_{i}, M_{i}\right)$, this end of the bar is at the point of $\mathbb{R}^{d}$ with coordinates $\tilde{q}^{i}=M_{i} q^{i}+p_{i}$.

All this data must respect the conditions required by periodicity; i.e., when we act on a vertex $i$ by a period $\gamma \in \Gamma$, we have $\tau(\gamma i)=\pi(\gamma) \tau(i)$ and $q^{i}(e)=q^{\gamma i}(\gamma e)$, etc. The main definition can now be given.

A $d$-periodic body-and-bar framework is a connected $d$-periodic graph $(G, \Gamma)$ together with a presentation $(\tau, \pi, q)$ where all bars have nonzero lengths. 


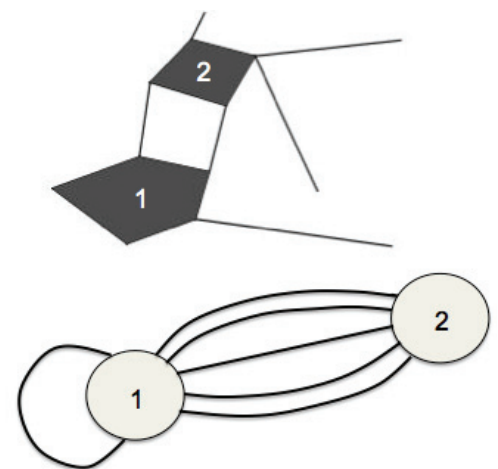

Fig. 7. Top: Representatives of orbits for bodies and bars for the periodic framework from Figure 6. Bottom: The quotient multigraph.

Realizations, configurations, rigid and flexible frameworks. Presentations which use the same attachment points $q$ (relative to the corresponding bodies) and induce the same system $\ell$ of bar lengths form the realization space for the data $(G, \Gamma, q, \ell)$.

Realizations that differ by an isometry of $\mathbb{R}^{d}$ will be considered as the same configuration. The configuration space of $(G, \Gamma, \tau, \pi, q)$ is defined as the quotient space of all realizations of $(G, \Gamma)$ which use the same attachment points $q$ (relative to the corresponding bodies) and induce the same system $\ell$ of bar lengths as $(G, \Gamma, \tau, \pi, q)$, modulo the group $E(d)$ of all isometries of $\mathbb{R}^{d}$. The deformation space of a periodic framework $(G, \Gamma, \tau, \pi, q)$ is the connected component of the corresponding configuration. When the deformation space consists of a single point, the framework is called rigid; otherwise it is flexible. An example of a flexible periodic framework is illustrated in Figure 6, with its quotient graph shown in Figure 7.

As in [1], this deformation theory has the following useful characteristics: (i) The periodicity group $\Gamma$ is part of the structure, and the deformations of a framework are those preserving this specified periodicity. (ii) The lattice of translations $\pi(\Gamma)$ representing $\Gamma$ is allowed to vary as the framework deforms. (iii) The realization space is the solution space of a finite algebraic system of quadratic equations. (iv) There is an infinitesimal deformation theory, obtained by differentiating the equations. This leads to the concept of infinitesimally rigid framework, characterized by a rigidity matrix of maximum rank.

In particular, standard arguments from algebraic geometry (inverse function theorem) can now be used to prove that infinitesimal rigidity implies rigidity.

Minimal rigidity. Following a heuristic which goes back to Maxwell [25], we do a quick calculation of the minimal number of parameters needed to specify a rigid framework. This gives us an indication of the number of bars we would expect in a minimally rigid graph. A vertex orbit requires $\left(\begin{array}{c}d+1 \\ 2\end{array}\right)$ parameters to be specified, and an additional $d^{2}$ parameters specify the periodicity lattice. The total number of variables in the algebraic system is thus $n\left(\begin{array}{c}d+1 \\ 2\end{array}\right)+d^{2}$. Each edge orbit leads to a constraint (equation) and is apt to eliminate one degree of freedom. Since we cannot eliminate the trivial isometries, we expect to have at most $n\left(\begin{array}{c}d+1 \\ 2\end{array}\right)+d^{2}-\left(\begin{array}{c}d+1 \\ 2\end{array}\right)=(n-1)\left(\begin{array}{c}d+1 \\ 2\end{array}\right)+d^{2}$ independent equations.

A minimally rigid d-periodic body-and-bar graph $(G, \Gamma)$ is a $d$-periodic graph with $m=(n-1)\left(\begin{array}{c}d+1 \\ 2\end{array}\right)+d^{2}$ which has an infinitesimally rigid presentation. 
For $n=2$ bodies in dimension 2 this number is 7 . Since the quotient graph from Figure 7 of the periodic framework from Figure 6 has $6<7$ bars, we expect the periodic framework to be flexible with one degree of freedom (1dof).

We now have all the ingredients to start developing the rigidity theory of periodic body-and-bar frameworks. The first step is to define and analyze the rigidity matrix associated to a generic framework.

The rigidity matrix. We express the rigidity matrix in terms of the following coordinates: We choose a basis $\lambda_{1}, \ldots, \lambda_{d}$ for the lattice of periods $\pi(\Gamma)$ and denote by $\Lambda$ the $d \times d$ matrix with columns $\lambda_{i}$; then we choose representatives for the $n$ equivalence classes of vertices modulo $\Gamma$ and consider the corresponding bodies as marked by $\left(p_{i}, M_{i}\right), i=1, \ldots, n$. We then choose an orientation of the edge set in the quotient graph $G / \Gamma$ and select representatives for the equivalence classes of edges modulo $\Gamma$ by the rule that all edge representatives originate at the already chosen vertex representatives. We remind the reader that the endpoints $q^{u}, q^{v}$ of an edge representative are fixed parameters, in the sense that the coordinates of these points, relative to the body to which they belong, are part of the specification of the bodyand-bar framework. The only restriction to their choice is that $\tilde{q}^{u} \neq \tilde{q}^{v}$, i.e., the two endpoints should not coincide (otherwise the (periodic) edge would have zero length). In order to keep the notation as simple as possible, we do not pursue the notational details related to marking distinctions between several bars possibly joining the same pair of bodies.

Since, by definition of representatives, one end (the origin) of any edge representative is on a body representative, the other end is on the corresponding representative translated by a period $\lambda_{e}$ for some $\lambda_{e} \in \pi(\Gamma)$. Using $\Lambda$, we can define a column vector $C(e)$ with integer entries $c_{i}, i=1, \ldots, d$, by $\lambda_{e}=\Lambda C(e)$.

Then, a bar constraint corresponding to an edge $e$ between bodies $i$ and $j$, with endpoints $q^{i}(e)$, respectively, $q^{j}(e)$, on these two bodies, takes the form

$$
\left\|\Lambda C(e)+M_{j} q^{j}(e)-M_{i} q^{i}(e)+p_{j}-p_{i}\right\|^{2}=\ell_{e}^{2} .
$$

There are $m$ such constraints, one for each edge $e$ from the set $E / \Gamma$ of edge representatives.

We will eliminate the equivalence under rigid motions by assuming that the first representative body is fixed, with $\left(p_{1}, M_{1}\right)=\left(0, I_{d}\right)$.

We note here that for an orthogonal matrix $M$, an "infinitesimal variation" $\dot{M}$ takes the form $\dot{M}=M A$, with $A$ skew-symmetric. Indeed, a differentiable curve $M(t)$ through $M$ (i.e., with $M(0)=M)$ in $S O(d)$ can be given in the form $M(t)=M L(t)$, with $L(t) \in S O(d)$ and $L(0)=I_{d}$, and hence

$$
\dot{M}=\frac{d M}{d t}(0)=M \frac{d L}{d t}(0), \text { with } \frac{d L}{d t}(0)=A \text { skew-symmetric. }
$$

Thus, if we differentiate the system at $\left(p_{2}, M_{2}\right), \ldots,\left(p_{n}, M_{n}\right), \Lambda$, we obtain a linear system in infinitesimal variations $\left(\dot{p}_{i}, A_{i}\right), i=2, \ldots, n$, and $\dot{\Lambda}$ with rows

$$
\left\langle\dot{\Lambda} c+M_{j} A_{j} q^{j}-M_{i} A_{i} q^{i}+\dot{p}_{j}-\dot{p}_{i}, \Lambda c+M_{j} q^{j}-M_{i} q^{i}+p_{j}-p_{i}\right\rangle=0,
$$

where we have dropped the notational dependency on $e$ and used indices $i, j$ for the chosen body representatives, with $M_{1}=I_{d}, p_{1}=0$, and $A_{1}=0$. The rigidity matrix is the matrix of this linear system (for some specific ordering of the unknowns).

Normalized coordinates. Given any $d$-periodic body-and-bar framework, the manner of marking chosen representatives in each vertex orbit by Cartesian frames 

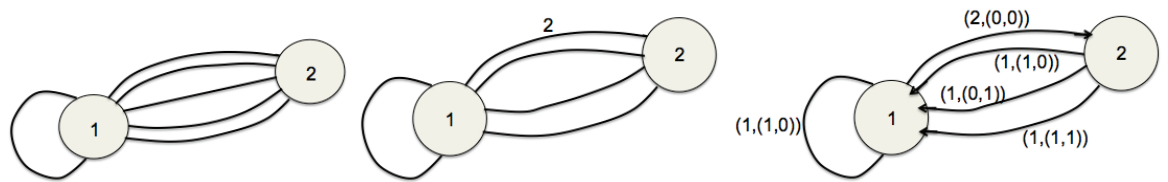

FIG. 8. Left: The basic quotient graph $G / \Gamma$. Center: The quotient graph with multiplicities $\mathcal{G} / \Gamma$. Right: The labeled and oriented quotient multigraph with multiplicities, equivalent with $(G, \Gamma)$.

$\left(p_{i}, M_{i}\right)$ is also a matter of choice, and we may adopt the normalization which has all $p_{i}=0$ and $M_{i}=I_{d}$. The rigidity matrix is then the matrix of the linear system in $\dot{p}_{i}, A_{i}=-A_{i}^{t}$, and $\dot{\Lambda}$ :

$$
\left\langle\dot{\Lambda} c+A_{j} q^{j}-A_{i} q^{i}+\dot{p}_{j}-\dot{p}_{i}, \Lambda c+q^{j}-q^{i}\right\rangle=0,
$$

where the edge vectors $\Lambda c+q^{j}-q^{i}$ run over all edge representatives.

3. Minimal rigidity. As already observed, up to Euclidean rigid motions, a periodic body-and-bar framework is described by $\left(\begin{array}{c}d+1 \\ 2\end{array}\right)(n-1)+d^{2}$ parameters. A bar equivalence class imposes a single constraint on these parameters, and hence rigidity requires at least $\left(\begin{array}{c}d+1 \\ 2\end{array}\right)(n-1)+d^{2}$ constraints. A $d$-periodic body-and-bar graph $(G, \Gamma)$, with $n=|E / \Gamma|$ and $m=|E / \Gamma|=\left(\begin{array}{c}d+1 \\ 2\end{array}\right)(n-1)+d^{2}$, is minimally rigid if it has a placement as a $d$-periodic body-and-bar framework in $\mathbb{R}^{d}$ with a rigidity matrix of maximal rank $m$. In this section we characterize these graphs in terms of a sparsity condition on their quotient graphs.

Liftings and marked quotient graphs. The problem of characterizing the structure of minimally rigid periodic graphs can be formulated at three distinct levels, illustrated in Figure 8. The most discerning and demanding level concerns the periodic graph $(G, \Gamma)$. Two other less discerning levels focus only on the quotient multigraph $G / \Gamma$, with or without some additional lifting information retained.

The information lost upon passing from the periodic framework $(G, \Gamma)$ to the quotient graph $G / \Gamma$ is the lifting of the edges to specific vertex representatives of the same orbit. This information can be retained in the following form. We fix a basis of $\Gamma$, that is, an isomorphism $\Gamma \approx Z^{d}$. Then we choose vertex representatives and an orientation of the quotient graph. By using edge representatives which originate at the chosen vertex representatives, we have a well defined lifting function $C: E / \Gamma \rightarrow Z^{d}$ which allows retrieval of $(G, \Gamma)$ from $G / \Gamma$. When several bars connect the same bodies, we can retain this information by joining the vertex representatives with a single edge, marked with a number indicating the edge multiplicity, as in Figure 8(center). This "multigraph with multiplicities" is denoted by $\mathcal{G} / \Gamma$. Combining the edge multiplicities with the lifting function yields a labeled multigraph with multiplicities, such as illustrated in Figure 8(right). This contains all the information needed to reconstruct $(G, \Gamma)$.

Remarks. What we call here a lifting appears in the literature under different names: [30] uses gain graph and [24] refers to the marks as colors on the edges.

An invariant way for describing the lifting data can be given in terms of the first homology group (with integer coefficients) $H_{1}(G / \Gamma):=H_{1}(G / \Gamma, Z)$, where $G / \Gamma$ is now seen as the topological space corresponding to the one-dimensional $\mathrm{CW}$-complex defined by the quotient graph [20]. With $G$ considered in the same topological perspective, we have a (connected) Abelian covering $G \rightarrow G / \Gamma$ with covering group $\Gamma$, and this is equivalent to a surjective group homomorphism $H_{1}(G / \Gamma) \rightarrow \Gamma$. 
In the rest of this paper, we give a complete characterization of $(G, \Gamma)$ for the case of a single body $n=1$, and a characterization in terms of the quotient graph $G / \Gamma$ for arbitrary $n$.

The $n=1$ case. The characterization of minimal rigidity in terms of $G / \Gamma$ is trivial for $n=1$, since the only condition about the quotient graph is that of having $d^{2}$ loops. This is enough for finding liftings which have infinitesimally rigid framework presentations. We address here the more refined questions related to $(G, \Gamma)$ and $\mathcal{G} / \Gamma$, assuming $m=d^{2}$. The quotient multigraph with multiplicities $\mathcal{G} / \Gamma$ has a single vertex and a number of loops with multiplicities $k_{i}, i=1, \ldots, \ell$, with $\sum_{i=1}^{\ell} k_{i}=d^{2}$. Minimal rigidity in this setting is equivalent with the condition that all multiplicities $k_{i}$ be at most $d$. This will follow from the solution of the sharper problem about the structure of $(G, \Gamma)$.

Recalling that $n=1, m=d^{2}$ and that we eliminate the equivalence under rigid motions by fixing $\left(p_{1}, M_{1}\right)=\left(0, I_{d}\right)$, we obtain from $(2.2)$ a rigidity matrix with $d^{2}$ rows of the form $C(e) \otimes\left[\Lambda C(e)+\left(q^{j}(e)-q^{i}(e)\right)\right]$, where the tensor notation stands for a listing of all the $d^{2}$ products of the $d$ components of the two indicated vectors. Since the endpoints of bars can be chosen at will, the edge vectors $\Lambda C(e)+\left(q^{j}(e)-q^{i}(e)\right)$ are arbitrary (nonzero) vectors, say $h=h(e)$. With simplified notation $c_{i}$ for the coordinates in $C(e)$, such a row can be presented as

$$
\left[h_{1}\left(c_{1} c_{2} \ldots c_{d}\right), h_{2}\left(c_{1} c_{2} \ldots c_{d}\right), \ldots, h_{d}\left(c_{1} c_{2} \ldots c_{d}\right)\right] .
$$

In this form we recognize a union operation on $d$ copies of a linear matroid.

THEOREM 3.1. Let $(G, \Gamma)$ be a d-periodic body-and-bar graph with $n=|V / \Gamma|=1$ and $m=|E / \Gamma|=d^{2}$. Then $(G, \Gamma)$ has infinitesimally rigid framework presentations in $\mathbb{R}^{d}$ if and only if, for any set of equivalence classes of edges $F \subset E / \Gamma$, we have

$$
|F| \leq d \cdot \operatorname{dim}(\operatorname{span} C(F)),
$$

where $C(F)=\{C(e): e \in F\}$.

Proof. The necessity of (3.1) follows directly from the form, described above, of the row constraints. The sufficiency follows from results in matroid theory, which will be used again in arguments for subsequent statements. Specifically, one can easily check that (3.1) holds for every $F \subset E / \Gamma$ if and only if $|E / \Gamma|=\min \{|F|+d \cdot \operatorname{dim}(\operatorname{span} C(F)) \mid$ $F \subset E / \Gamma\}$. The latter condition is equivalent to saying that $E / \Gamma$ is independent in the union of $d$ copies of the matroid associated to the rank function $\operatorname{dim}(\operatorname{span} C(F))$. This is because $\min \{|F|+d \cdot \operatorname{dim}(\operatorname{span} C(F)) \mid F \subset E / \Gamma\}$ is equal to the rank of $E / \Gamma$ in the union of the $d$ copies by the Nash-Williams matroid union theorem [29] (see also section 12.2 of [28] for more details). Hence a basis of the former is a disjoint union of bases in the latter which yield the maximal rank of the rigidity matrix.

This proof also implies our earlier claim that a quotient graph $\mathcal{G} / \Gamma$ comes from a minimally rigid $(G, \Gamma)$ if and only if all multiplicities $k_{i}, i=1, \ldots, \ell$, are at most $d$. Indeed, any choice of lifting function $C: E / \Gamma \rightarrow Z^{d}$, with image consisting of classes of at most $d$ vectors (corresponding to the given multiplicities) and with representatives in general position, will satisfy the criterion of Theorem 3.1.

4. The quotient graph. In this section we present the main theoretical result. We assume that $m=\left(\begin{array}{c}d+1 \\ 2\end{array}\right)(n-1)+d^{2}$ and characterize the quotient graphs $G / \Gamma$ which are obtained from minimally rigid $d$-periodic body-and-bar graphs $(G, \Gamma)$.

Graph sparsity. We say that a multigraph $G=(V, E)$ (possibly with loops) is $(a, b)$-sparse, or has an $-b$ sparsity type, if any subset of $n^{\prime} \leq|V|$ vertices spans at 
most $a n^{\prime}-b$ edges. When equality holds we have a tight (sub)graph. See [22] for a comprehensive treatment of this kind of graph sparsity.

When $0 \leq b<2 a$, the family of $(a, b)$-sparse graphs on a fixed number of vertices $n$ forms a matroid. When $b=a$, this matroid is the union of $b$ graphic (tree) matroids. When $b=0$, it is the union of $a$ bicycle matroids, whose independent sets are the $(1,0)$-sparse graphs (also known in the literature as map-graphs or pseudoforests, and whose circuits are called bicycles, as they are connected graphs with exactly two cycles). We also obtain a matroid when $b<0$ : as shown in Corollary 4.2 below, it is the union of the $(a, 0)$-sparsity matroid and the uniform matroid on $b$ elements, or, equivalently, it is any graph which has $a n+b$ edges and contains an $(a, 0)$-sparse subgraph with $n$ vertices.

We characterize now the quotient graphs of periodic minimally rigid graphs in terms of matroid unions of graph sparsity matroids. For easy reference, we reproduce here our main result stated in the introduction.

Theorem 1.1 (restated). A multigraph with $n$ vertices and $m=\left(\begin{array}{c}d+1 \\ 2\end{array}\right)(n-1)+d^{2}$ edges is the quotient graph of an infinitesimally rigid periodic body-and-bar framework in $\mathbb{R}^{d}$ if and only if it satisfies one and hence both of the following equivalent conditions:

(i) it decomposes into a disjoint union of two graphs on the full set of $n$ vertices, one with $d n-d$ edges and sparsity type, and the other with $\left(\begin{array}{l}d \\ 2\end{array}\right) n+\left(\begin{array}{c}d+1 \\ 2\end{array}\right)$ edges and sparsity type;

(ii) it contains the union of two edge-disjoint graphs on the full set of $n$ vertices, one with $d n-d$ edges and sparsity type, and the other with $\left(\begin{array}{l}d \\ 2\end{array}\right) n$ edges and sparsity type.

As shown in [2], graphs with $d n-d$ edges on $n$ vertices and with $d n-d$ sparsity type are minimally rigid graphs for the problem of rigidly connecting with bars a set of $n$ translated bodies (i.e., parallel Cartesian frames). Thus, the presence of a $d n-d$ sparse subgraph is related to the fact that vertex orbits (when "frozen solid" together into a body) provide a system of $n$ translated bodies which has to become rigid.

Proof of sufficiency. We begin with some combinatorial background about sparsity of type $a n+b$, with $a$ and $b$ nonnegative integers. Again, in this discussion, graphs may have multiple edges and loops.

Loop breaking. When a graph has loops, we shall describe as loop breaking the operation of replacing a loop by an edge connecting the loop vertex with some other vertex of the graph. It is immediate that loop breaking preserves $a n+b$ sparsity. The following lemma is the relevant converse.

Lemma 4.1. Let $G$ be a graph with an $+b$ edges on $n$ vertices, with $a$ and $b$ nonnegative integers. Suppose $G$ is an $+b$ sparse, that is, any subset of $n^{\prime}$ vertices has at most $a n^{\prime}+b$ edges between them. Then, there is a graph $\tilde{G}$, made of $a n+b$ loops on $n$ vertices, which is an $+b$ sparse and yields $G$ after an adequate sequence of sparsity-preserving loop breaking steps.

Proof. We claim that we can always replace an edge of $G$ between vertices $u$ and $v$ by a loop at one of these two vertices so that $a n+b$ sparsity is preserved. Suppose this were not the case. Then there are subsets of vertices $U$ and $V$, with $u$ in $U$ but not in $V$ and $v$ in $V$ but not in $U$, with $a|U|+b$ edges in $U$ and $a|V|+b$ edges in $V$. Since the union $U \cup V$ has at most $a|U \cup V|+b$ edges, the intersection $U \cap V$ must be nonvoid and tight. Even so, the union $U \cup V$ has already $a|U \cup V|+b$ edges without counting the edge we started with. This contradiction proves the claim, and 
the lemma follows by iteration. Note that the resulting graph $\tilde{G}$ must have at least $a$ loops at each vertex.

COROLlary 4.2. With the notation of the above lemma, $G$ is "an $+b$ " sparse if and only if it contains a spanning subgraph of sparsity type "an," with a number of edges $m=$ an on the $n$ vertices.

We show now that a loop breaking operation may be performed on minimal infinitesimally rigid frameworks. Let us assume that such a framework has a loop at vertex $j$ in the quotient graph $G / \Gamma$. Using normalized coordinates as in (2.3), the loop corresponds to an equation of the form

$$
\left\langle\dot{\Lambda} c+A_{j}\left(q^{j}-q^{\prime j}\right), \Lambda c+q^{j}-q^{\prime j}\right\rangle=0,
$$

which involves only the difference $q^{j}-q^{\prime j}$. This allows the assumption $q^{\prime j}=0$ for the origin of the bar. We are going to replace this loop at vertex $j$ of $G / \Gamma$ by an edge from vertex $i$ to vertex $j$. The corresponding bar has one endpoint at the origin of the body representative indexed by $i$ and the other endpoint at $k q^{j}$ on the body representative indexed by $j$ translated by period $\Lambda k c$, for some integer $k$. This gives an equation of the form

$$
\left\langle\dot{\Lambda} k c+A_{j} k q^{j}+\dot{p}_{j}-\dot{p}_{i}, \Lambda k c+k q^{j}\right\rangle=0,
$$

which, divided by $k^{2}$, takes the form

$$
\left\langle\dot{\Lambda} c+A_{j} q^{j}+\frac{1}{k}\left(\dot{p}_{j}-\dot{p}_{i}\right), \Lambda c+q^{j}\right\rangle=0 .
$$

With all other rows unchanged, the rigidity matrices for the two frameworks involve only the exchange of row (4.1), where $q_{j}^{\prime}=0$, with row (4.3) and for a sufficiently large integer $k$, the rank will not be affected. This shows that loop breaking operations can be performed on quotients of minimally rigid graphs with preservation of minimal rigidity.

The sufficiency proof follows from these results. Indeed, if $G / \Gamma$ has edges separated into a $d n-d$ tight sparse graph and a $\left(\begin{array}{l}d \\ 2\end{array}\right) n+\left(\begin{array}{c}d+1 \\ 2\end{array}\right)$ tight sparse graph, we can produce minimally rigid $d$-periodic graphs with that quotient by a two-step procedure. First, we use the $d n-d$ sparse subgraph, but replace the remainder graph with a graph made only of loops, as guaranteed by Lemma 4.1. Infinitesimally rigid frameworks corresponding to this quotient structure can be constructed in a natural way, as indicated immediately below. Then, as a second step, we break the appropriate loops and obtain the initial graph while maintaining minimal rigidity.

We first remark that a bar that fixes a period, i.e., a bar that joins two copies of the same body $k$ (they differ by a period) and whose endpoints $q_{i}$ and $q_{i}$ (relative to the reference body) are identical $\left(q_{i}=q_{j}\right)$, induces a loop at vertex $k$ in the quotient graph.

When the $\left(\begin{array}{c}d \\ 2\end{array}\right) n+\left(\begin{array}{c}d+1 \\ 2\end{array}\right)$ tight sparse subgraph is made entirely of loops, natural liftings of $G / \Gamma$ to minimally rigid $(G, \Gamma)$ are constructed as follows. First, we use $\left(\begin{array}{c}d+1 \\ 2\end{array}\right)$ loops to make rigid the lattice of periods, as in $[1,2]$.

We distribute them so that exactly $\left(\begin{array}{l}d \\ 2\end{array}\right)$ loops remain for bar assignment at each vertex class. Now in each vertex orbit we place the available $\left(\begin{array}{l}d \\ 2\end{array}\right)$ classes of bars so that the whole body-and-bar orbit becomes a single rigid body. Thus, with the $\left(\begin{array}{l}d \\ 2\end{array}\right) n+\left(\begin{array}{c}d+1 \\ 2\end{array}\right)$ loop subgraph we have turned the system into $n$ parallel bodies, and now the $d n-d$ 
sparse subgraph is precisely what we need to turn this system into a single rigid body, as in [2] (Theorem 2).

Proof of necessity. We consider the $m \times u$ rigidity matrix of an infinitesimally rigid periodic body-and-bar framework in $\mathbb{R}^{d}$. The number of rows $m=\left(\begin{array}{c}d+1 \\ 2\end{array}\right)(n-1)+d^{2}$ is the number of edge orbits, and the number of columns $u=\left(\begin{array}{c}d+1 \\ 2\end{array}\right) n+d^{2}=m+\left(\begin{array}{c}d+1 \\ 2\end{array}\right)$ is the number of unknowns in the linear constraint system for infinitesimal variations. By assumption, the rows are linearly independent, and the $\left(\begin{array}{c}d+1 \\ 2\end{array}\right)$-dimensional kernel of our rigidity matrix consists precisely of the trivial infinitesimal rigid motions.

When we consider some subset of rows, denoted say by $F$, we have $|F|=r k(F)=$ $u-\operatorname{dim}(\operatorname{Ker}(F))$. We denote by $n_{F}$ the number of vertex orbits incident to the given edge orbits and by $\omega_{F}$ the number of connected components of $F$ as a set of edges in the quotient graph. We observe that

$$
\operatorname{dim}(\operatorname{Ker}(F)) \geq\left(\begin{array}{c}
d+1 \\
2
\end{array}\right)+\left(n-n_{F}\right)\left(\begin{array}{c}
d+1 \\
2
\end{array}\right)+d\left(\omega_{F}-1\right),
$$

where the numbers on the right-hand side count the following independent infinitesimal deformations respecting the constraint system $F$ : trivial infinitesimal rigid motions, arbitrary infinitesimal deformations for the body representatives for nonincident vertices, and relative infinitesimal translations of the connected components in $F$. Thus, we have a necessary edge sparsity condition:

$$
|F|=u-\operatorname{dim}(\operatorname{Ker}(F)) \leq d\left(n_{F}-\omega_{F}\right)+\left(\begin{array}{l}
d \\
2
\end{array}\right) n_{F}+\left(\begin{array}{c}
d+1 \\
2
\end{array}\right) .
$$

Written in this form, the right-hand side becomes very suggestive from the point of view of matroid theory [28]. Indeed, if we consider a ground set of sufficiently many edges and loops on $n$ vertices (so that any graph with $m$ edges can be found as a subgraph), then the function defined by the right-hand side is an increasing submodular function with values in the nonnegative integers and determines a matroid which is, by the matroid union theorem, the union of $d$ copies of the graphic matroid determined by $f_{1}(F)=n_{F}-\omega_{F}$, and then $\left(\begin{array}{l}d \\ 2\end{array}\right)$ copies of the bicycle matroid determined by $f_{2}(F)=n_{F}$ and the uniform matroid determined by $f_{3}(F)=\left(\begin{array}{c}d+1 \\ 2\end{array}\right)$.

A basis in a union of $d$ graphic matroids is a union of $d$ spanning trees, and this is precisely what $d n-d$ tight sparse graphs are, while $\left(\begin{array}{l}d \\ 2\end{array}\right) n+\left(\begin{array}{c}d+1 \\ 2\end{array}\right)$ tight sparse graphs give the bases of the remaining union. Thus, the $m$ edges corresponding to our maximal rank rigidity matrix have the claimed decomposition.

This completes the proof of our main result, Theorem 1.1.

5. Mixed plate-and-bar frameworks. The result proven above for minimally rigid $d$-periodic body-and-bar frameworks is obviously related to the characterization obtained in [2] for minimally rigid $d$-periodic bar-and-joint graphs. Actually, these two types of periodic framework structures may be seen as the extreme cases $k=d$, respectively $k=0$, of the family obtained by articulating with bars $k$-dimensional plates in $\mathbb{R}^{d}$, with $0 \leq k \leq d$. A plate is understood in this context as a rigid object marked by a $k$-frame in $\mathbb{R}^{\frac{}{d}}$. An example is illustrated in Figure 9 through rod-and-bar frameworks in three dimensions, in the finite (nonperiodic) case.

Remark. The distinction between articulations made of $(d-1)$-dimensional plates, also called panels, and those made of bodies, which are $d$-dimensional plates, is that bars have to be attached at points of the panel. Otherwise, any $(d-1)$-frame has a unique extension to a $d$-frame with standard orientation. 


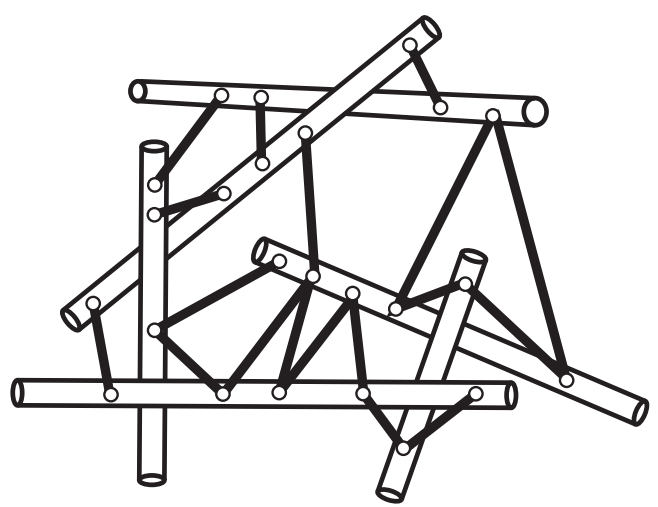

FIG. 9. A finite rod-and-bar framework where each plate is one-dimensional.

We observe that, since $k$-frames in $\mathbb{R}^{d}$ can be parametrized by $S E(d) / S O(d-k)$, the periodic setting with variable period lattice will require for minimal rigidity the relation

$$
\begin{gathered}
m=\left[d(k+1)-\left(\begin{array}{c}
k+1 \\
2
\end{array}\right)\right] n+d^{2}-\left(\begin{array}{c}
d+1 \\
2
\end{array}\right) \\
=(d n-d)+\left(d k-\left(\begin{array}{c}
k+1 \\
2
\end{array}\right)\right) n+\left(\begin{array}{c}
d+1 \\
2
\end{array}\right) .
\end{gathered}
$$

The extended version of the main theorem is that a quotient graph comes from a minimally rigid $d$-periodic plate-and-bar framework (with all plates of dimension $k$ ) if and only if it contains the disjoint union of two graphs on the full set of $n$ vertices, one with $d n-d$ edges and sparsity type, and the other with $\left(d k-\left(\begin{array}{c}k+1 \\ 2\end{array}\right)\right) n$ edges and sparsity type. Note that, as in Theorem 1.1(ii), there is no condition on the remaining $\left(\begin{array}{c}d+1 \\ 2\end{array}\right)$ edges of $G / \Gamma$.

We may consider a more general setting by mixing various types of plates. The associated $d$-periodic graph $(G, \Gamma)$ will have vertices labeled according to the dimension of the plate they represent. This leads to a quotient graph $G / \Gamma$ with labels, or weights $k_{v} \in\{0,1, \ldots, d\}$ for each $v \in V / \Gamma$. With vertex orbits labeled from 1 to $n$, we write $k_{i}, i=1, \ldots, n$, for the corresponding (dimensional) weights. We also use $\omega_{F}$ for the number of connected components of $F$ as a set of edges in the quotient graph. With these notations, we can now state the following general characterization of minimal rigidity for plate-and-bar frameworks.

TheOREM 5.1. A vertex weighted multigraph (with weights $k_{i}$ as above), with

$$
m=d(n-1)+\sum_{i=1}^{n}\left[d k_{i}-\left(\begin{array}{c}
k_{i}+1 \\
2
\end{array}\right)\right]+\left(\begin{array}{c}
d+1 \\
2
\end{array}\right),
$$

is the quotient graph of an infinitesimally rigid mixed plate-and-bar periodic framework in $\mathbb{R}^{d}$ if and only if it satisfies the sparsity condition

$$
|F| \leq d\left(n_{F}-\omega_{F}\right)+\sum_{v \in V_{F}}\left[d k_{v}-\left(\begin{array}{c}
k_{v}+1 \\
2
\end{array}\right)\right]+\left(\begin{array}{c}
d+1 \\
2
\end{array}\right)
$$

for every nonempty set $F$ of edges in the multigraph, where $V_{F}$ is the set of vertices incident to $F$. 
Remark. The edge sparsity formula (5.1) replaces formula (4.5) of the "pure" top case $k_{v}=d$. Its role and combinatorial unfolding can be treated similarly. Let $k_{v}^{\prime}=\left[d k_{v}-\left(\begin{array}{c}k_{v}+1 \\ 2\end{array}\right)\right]$. The function defined by the right-hand side of (5.1) determines a matroid which is the union of $d$ copies of the graphic matroid, the uniform matroid with rank $\left(\begin{array}{c}d+1 \\ 2\end{array}\right)$, and the matroid determined by $f_{4}(F)=\sum_{v \in V_{F}} k_{v}^{\prime}$.

The loop breaking scenario used in section 3 remains valid for the mixed case and shows in particular that any basis of the matroid determined by $f_{4}$ is obtained from the graph with $k_{v}^{\prime}$ loops at each vertex of $V / \Gamma$ by loop breaking.

In this sense, archetypal infinitesimally rigid frameworks are obtained by fixing the periodicity lattice through $\left(\begin{array}{c}d+1 \\ 2\end{array}\right)$ bars which are periods; then the $k_{v}^{\prime}$ loops at the vertex orbit of $v$ are used to eliminate the relative motion of the $k_{v}$-frame with respect to the lattice and turn the whole orbit of $v$ into a rigid body. Finally, the $n \Gamma$-orbits are a system of translated bodies which is rendered rigid by using the remaining $d$ spanning trees. An alternative, more detailed argument for the proof of Theorem 5.1 is the following.

Proof. The necessity part follows from the same argument as in Theorem 1.1 by counting the number of independent motions. We show that sufficiency also follows from the loop breaking argument given in Theorem 1.1.

We first provide the corresponding combinatorial part. Let $k_{v}^{\prime}=\left[d k_{v}-\left(\begin{array}{c}k_{v}+1 \\ 2\end{array}\right)\right]$ for simplicity. In the necessity proof of Theorem 1.1, we have seen how the matroid determined by the corresponding count is decomposed for body-and-bar frameworks. In the present case, the function defined by the right-hand side of (5.1) is also an increasing submodular function with values in nonnegative integers and it determines a matroid which is the union of $d$ copies of the graphic matroid, the uniform matroid with rank $\left(\begin{array}{c}d+1 \\ 2\end{array}\right)$, and the matroid determined by $f_{4}(F)=\sum_{v \in V_{F}} k_{v}^{\prime}$ which is also an increasing submodular function with values in nonnegative integers.

To further decompose the last matroid, let $V_{\ell}=\left\{v \in V: k_{v} \geq \ell\right\}$ for $\ell=0, \ldots, d$ and define $g_{\ell}(F)=\left|V_{\ell} \cap V_{F}\right|$. Observe that $f_{4}=\sum_{\ell=1}^{d-1}(d-\ell) g_{\ell}$, and hence the matroid determined by $f_{4}$ can be further decomposed into $\left(\begin{array}{l}d \\ 2\end{array}\right)$ matroids. This gives us a decomposition of $\sum_{v \in V} k_{v}^{\prime}$ edges into $\left(\begin{array}{l}d \\ 2\end{array}\right)$ subsets $F_{k \ell}$ for $1 \leq \ell \leq d-1$ and $1 \leq k \leq d-\ell$ such that $F_{k \ell}$ is a pseudoforest spanning only vertices of $V_{\ell}$ (i.e., $F_{k \ell}$ is an edge set on $V_{\ell}$ with $\left|F_{k \ell}\right|=\left|V_{\ell}\right|$ such that each connected component contains exactly one cycle). Each $F_{k \ell}$ can be oriented so that each vertex in $V_{\ell}$ has in-degree exactly one, and this orientation implies the following lemma, which supplies the converse direction of loop breaking operations.

Lemma 5.2. Let $G$ be a graph with $\sum_{v \in V} k_{v}^{\prime}$ edges on $n$ vertices, with $k_{v}^{\prime}$ nonnegative integers. Suppose $|F| \leq \sum_{v \in V_{F}} k_{v}^{\prime}$ for $F \subseteq E$. Then, there is a graph $\tilde{G}$, made of $k_{v}^{\prime}$ loops for each vertex $v \in V$, which yields $G$ after an adequate sequence of loop breakings.

We thus convert $G / \Gamma$ into a graph which consists of a $d n-d$ tight sparse subgraph, $\left(\begin{array}{c}d+1 \\ 2\end{array}\right)$ edges, and $k_{v}^{\prime}$ loops for each $v \in V$. As before, if the $\left(\begin{array}{c}d+1 \\ 2\end{array}\right)$ are further converted into $\left(\begin{array}{c}d+1 \\ 2\end{array}\right)$ loops, then we have the base graph. Of all these loops, $\left(\begin{array}{c}d+1 \\ 2\end{array}\right)$ of them are used to make rigid the lattice of periods. In each vertex orbit $v$, we place $k_{v}^{\prime}$ classes of bars, corresponding to $k_{v}^{\prime}$ loops attached to $v$, so that the whole $k_{v}$-dimensional plate-and-bar orbit becomes a single body. Thus, using the $\sum_{v \in V} k_{v}^{\prime}+\left(\begin{array}{c}d+1 \\ 2\end{array}\right)$ loops, we have turned the system into $n$ parallel bodies, and the remaining $d n-d$ sparse subgraph makes this system into a single rigid body, as in [2].

The proof is completed by performing a sequence of loop breaking operations, as we have shown in Theorem 1.1. In the realization given in the previous paragraph, we 
may assume that each $k_{v}$-dimensional plate is the one spanned by the first $k_{v}$ column vectors of $I_{d}$. Then the rigidity matrix is written in the same form:

$$
\left\langle\dot{\Lambda} c+A_{j} q^{j}-A_{i} q^{i}+\dot{p}_{j}-\dot{p}_{i}, \Lambda c+q^{j}-q^{i}\right\rangle=0
$$

over all edge representatives, where $A_{i}$ is a skew-symmetric matrix with $k_{v}^{\prime}$ nonzero entries. Thus we can apply exactly the same argument as in the proof of Theorem 1.1 to show that a loop breaking operation preserves infinitesimal rigidity.

6. On minimal rigidity in terms of $(\boldsymbol{G}, \boldsymbol{\Gamma})$. The case $n=1$ in section 3 already illustrated the contrast between an answer given in terms of $G / \Gamma$, which allows a generic lifting of edges to a $d$-periodic covering graph, and an answer given in terms of the periodic graph $(G, \Gamma)$ itself: Theorem 3.1. We discuss here several aspects which must be implicated when minimal rigidity is addressed directly on $(G, \Gamma)$. We assume $G$ connected and $m=\left(\begin{array}{c}d+1 \\ 2\end{array}\right)(n-1)+d^{2}$.

We reviewed in section 3 the fact that the covering $G \rightarrow G / \Gamma$ with covering group $\Gamma$ can be equivalently described through $G / \Gamma$ and a surjective group homomorphism $H_{1}(G / \Gamma) \rightarrow \Gamma$. For a given subset $F$ of edges in $G / \Gamma$, we shall consider the following numbers or invariants defined in terms of the quotient graph and the surjective homomorphism describing its covering $(G, \Gamma): n_{F}, \omega_{F}, d_{F}, d_{F^{\prime}}$, where $F^{\prime}$ denotes a connected component of $F$ when considered as a topological subspace of $G / \Gamma$.

We use the following notation: $n_{F}$ is the number of vertices in $G / \Gamma$ which are incident to edges in $F$, and $\omega_{F}$ is the number of connected components of $F$ as a topological subspace $F \subset G / \Gamma$.

For each connected component $F^{\prime} \subset F \subset G / \Gamma$ we consider the induced maps

$$
H_{1}\left(F^{\prime}\right) \rightarrow H_{1}(F) \rightarrow H_{1}(G / \Gamma) \rightarrow \Gamma
$$

and define $d_{F}$ as the rank of the image of $H_{1}(F)$ in $\Gamma$, while $d_{F^{\prime}}$ will be the rank of the image of $H_{1}\left(F^{\prime}\right)$ in $\Gamma$. Obviously $d_{F^{\prime}} \leq d_{F}$.

Now we formulate an estimate for the dimension of the kernel of the matrix with row constraints determined by $F$ :

$$
\begin{gathered}
\operatorname{dim}(\operatorname{Ker}(F)) \geq\left(\begin{array}{c}
d+1 \\
2
\end{array}\right)+\left(n-n_{F}\right)\left(\begin{array}{c}
d+1 \\
2
\end{array}\right)+d\left(\omega_{F}-1\right) \\
+\sum_{F^{\prime}}\left(\begin{array}{c}
d-d_{F^{\prime}} \\
2
\end{array}\right)+\left[\left(\begin{array}{c}
d+1 \\
2
\end{array}\right)-\left(\begin{array}{c}
d_{F}+1 \\
2
\end{array}\right)\right],
\end{gathered}
$$

where $F^{\prime}$ is taken over all connected components of $F$. We remark that the new term $\sum_{F^{\prime}}\left(\begin{array}{c}d-d_{F^{\prime}} \\ 2\end{array}\right)$ appears on the right-hand side because of the fact that, if liftings over a connected component $F^{\prime}$ involve only a subspace of the lattice of periods, there are independent infinitesimal rotations in the orthogonal complement. Also, to see why the last term $\left(\begin{array}{c}d+1 \\ 2\end{array}\right)-\left(\begin{array}{c}d_{F}+1 \\ 2\end{array}\right)$ appears on the right-hand side, note that, when no bar constraints are present, each lattice has $\left(\begin{array}{c}d+1 \\ 2\end{array}\right)$ degrees of freedom up to isometries. Hence, if liftings over $F$ involve only a subspace of the lattice of periods, only at most $\left(\begin{array}{c}d_{F}+1 \\ 2\end{array}\right)$ degrees of freedom among them are eliminated.

With $|F|$ denoting the number of edges in $F$, we have

$$
|F| \leq d\left(n_{F}-\omega_{F}\right)+\left(\begin{array}{l}
d \\
2
\end{array}\right) n_{F}-\sum_{F^{\prime}}\left(\begin{array}{c}
d-d_{F^{\prime}} \\
2
\end{array}\right)+\left(\begin{array}{c}
d_{F}+1 \\
2
\end{array}\right) .
$$

Copyright $@$ by SIAM. Unauthorized reproduction of this article is prohibited. 

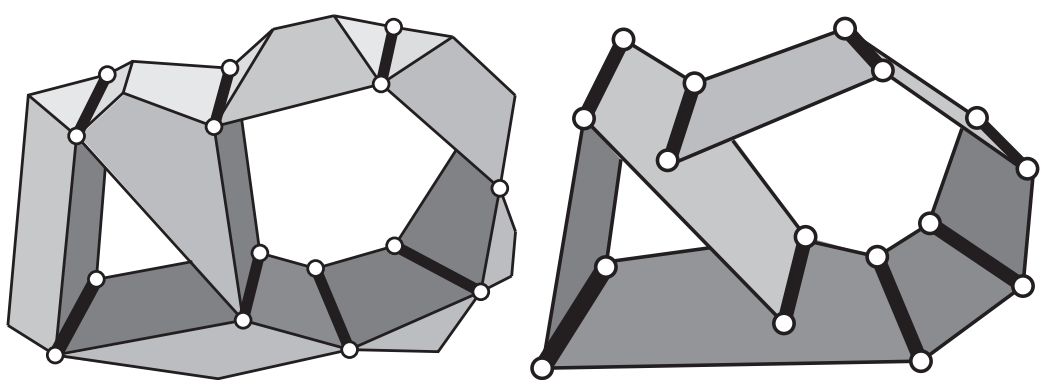

FIG. 10. Finite body-and-hinge and panel-and-hinge frameworks.

The question of whether this condition is also sufficient for a combinatorial characterization of minimal rigidity is left as an open problem. We note that for $n=1$, condition (6.3) becomes $|F| \leq d \cdot \operatorname{dim}(\operatorname{span} C(F))$, since $F$ is connected and $d_{F}=$ $\operatorname{dim}(\operatorname{span} C(F))$. We also remark that, in principle, the two-dimensional case $d=2$ may be approached by converting periodic body-and-bar frameworks into periodic bar-and-joint frameworks which can be tested by the criterion of [24]. However, the conversion process is not standard, as it involves choices of Laman graphs on each body between all anchor points of connecting bars.

7. Algorithms. We present here the algorithmic consequences of Theorem 1.1. Since our Maxwell-Laman characterization decomposes the matroids associated with minimal rigidity of periodic frameworks into smaller pieces of well-known (graphic and bicycle) matroids, we can simply apply a matroid partitioning algorithm (see, e.g., [10]) for checking whether a given quotient graph $G / \Gamma$ is realizable as a minimally rigid periodic framework.

However, we can give a better algorithm using condition (ii) of Theorem 1.1. It follows from a theorem of [14] that a graph is the disjoint union of a $d n-d$ tight subgraph and a $\left(\begin{array}{l}d \\ 2\end{array}\right) n$ tight subgraph if and only if it is a $\left(\begin{array}{c}d+1 \\ 2\end{array}\right) n-d$ tight graph. This type of sparsity is matroidal (i.e., the family of tight subgraphs provides the bases of a matroid on the edges of the complete graph), allowing for the use of the pebble game algorithms of [22]. Thus condition (ii) can be checked in $O\left(n^{2}\right)$ time. The same algorithm computes the degrees of freedom of a generic realization of a given quotient graph, where $m$ may not be exactly $\left(\begin{array}{c}d+1 \\ 2\end{array}\right)(n-1)+d^{2}$. This is done by first computing a maximum $\left(\begin{array}{c}d+1 \\ 2\end{array}\right) n-d$ sparse subgraph and then adding at most $\left(\begin{array}{c}d+1 \\ 2\end{array}\right)$ unused edges. The number of edges of the resulting graph gives the maximum size of independent equations, with its difference from $\left(\begin{array}{c}d+1 \\ 2\end{array}\right)(n-1)+d^{2}$ being the degree of freedom.

8. Conclusions. Motivated by computational studies of crystalline materials, we introduced periodic body-and-bar frameworks. We gave a combinatorial characterization of those which are generically minimally rigid, obtaining polynomial time algorithms for recognition and for computing flexibility parameters (degrees of freedom) of those which are generically flexible.

Other important molecular models are based on body-and-hinge frameworks, which allow rotational motions of rigid bodies connected through hinges, as in Figure 10. It is well known that the formal rigidity analysis of finite body-and-hinge structures can be reduced to body-and-bar frameworks by replacing a hinge with five bars. This reduction, when applied to the periodic case, would have to require that the endpoints of these five bars be placed on the same body and not lifted to some other periodic copy of it. Finding a suitable generic characterization for periodic body-and-hinge frameworks requires further investigation. 
We mention a short list of open problems: (a) characterize rigidity for body-andhinge frameworks, (b) characterize rigidity for body-and-pin periodic frameworks, and (c) verify whether the molecular conjecture [19] holds as well in the periodic case. Besides intrinsic theoretical interest, such results would have immediate applications, as these structures appear in modeling families of crystalline materials.

\section{REFERENCES}

[1] C. S. Borcea and I. Streinu, Periodic frameworks and flexibility, Proc. Roy. Soc. A, 466 (2010), pp. 2633-2649.

[2] C. S. Borcea And I. Streinu, Minimally rigid periodic graphs, Bull. Lond. Math. Soc., 43 (2011), pp. 1093-1103.

[3] C. S. Borcea And I. Streinu, Frameworks with crystallographic symmetry, Phil. Trans. R. Soc. A Math. Phys. Eng. Sci., 372 (2014), 20120143.

[4] J. H. Conway And N. J. A. Slonne, Sphere Packings, Lattices and Groups, 3rd ed., Grundlehren Math. Wiss. 290, Springer-Verlag, New York, 1999.

[5] O. Delgado-Friedrichs, Equilibrium placement of periodic graphs and convexity of plane tilings, Discrete Comput. Geom., 33 (2005), pp. 67-81.

[6] V. S. Deshpande, M. F. Ashby, And N. A. Fleck, Foam topology: Bending versus stretching dominated architectures, Acta Materialia, 49 (2001), pp. 1035-1040.

[7] G. Dolino, The $\alpha$-inc- $\beta$ transitions of quartz: A century of research on displacive phase transitions, Phase Transitions, 21 (1990), pp. 59-72.

[8] A. Donev and S. Torquato, Energy-efficient actuation in infinite lattice structures, J. Mech. Phys. Solids, 51 (2003), pp. 1459-1475.

[9] M. T. Dove, Theory of displacive phase transitions in minerals, American Mineralogist, 82 (1997), pp. 213-244.

[10] J. Edmonds, Minimum partition of a matroid into independent sets, J. Res. Nat. Bur. Standards Sect. B, 69 (1965), pp. 67-72.

[11] N. Fox, F. Jagodzinski, Y. Li, and I. Streinu, KINARI-web: A server for protein rigidity analysis, Nucleic Acids Research, 39 (2011), pp. W177-W183. Web server issue.

[12] A. L. Goodwin, Rigid unit modes and intrinsic flexibility of linearly bridged framework structures, Phys. Rev. B, 74 (2006), 134302.

[13] S. Guest And J. W. Hutchinson, On the determinacy of repetitive structures, J. Mech. Phys. Solids, 51 (2003), pp. 383-391.

[14] R. Haas, A. Lee, I. Streinu, And L. Theran, Characterizing sparse graphs by map decompositions, J. Combin. Math. Combin. Comput., 62 (2007), pp. 3-11.

[15] D. Hilbert and S. Cohn-Vossen, Geometry and the Imagination, Chelsea Publishing Company, New York, 1962.

[16] R. G. Hutchinson And N. A. Fleck, The structural performance of the periodic truss, J. Mech. Phys. Solids, 54 (2006), pp. 765-782.

[17] B. JACKSON AND T. JORDÁn, The generic rank of body-bar-and-hinge frameworks, European J. Combin., 31 (2009), pp. 574-588.

[18] V. Kapko, M. M. J. Treacy, M. F. Thorpe, and S. Guest, On the collapse of locally isostatic networks, Proc. Roy. Soc. A, 465 (2009), pp. 3517-3530.

[19] N. Katoh and S. Tanigawa, A proof of the molecular conjecture, Discrete Comput. Geom., 45 (2011), pp. 647-700.

[20] M. Kotani And T. Sunada, Standard realizations of crystal lattices via harmonic maps, Trans. Amer. Math. Soc., 353 (2001), pp. 1-20.

[21] G. Laman, On graphs and rigidity of plane skeletal structures, J. Engrg. Math., 4 (1970), pp. 331-340.

[22] A. Lee And I. Streinu, Pebble game algorithms and sparse graphs, Discrete Math., 308 (2008), pp. $1425-1437$.

[23] J. Lima-De-FARia, ED., Historical Atlas of Crystallography, Kluwer, Dordrecht, 1990.

[24] J. Malestein and L. Theran, Generic combinatorial rigidity of periodic frameworks, Adv. Math., 233 (2013), pp. 291-331.

[25] J. C. Maxwell, On the calculation of the equilibrium and stiffness of frames, Philosophical Magazine, 27 (1864), pp. 294-299.

[26] H. D. Megaw, Crystal structures: A working approach, W.B. Saunders Co., Philadelphia, 1973.

[27] M. O'Keeffe, M. Eddaoudi, H. Li, T. Reineke, and O. M. Yaghi, Frameworks for extended solids: Geometrical design principles, J. Solid State Chem., 152 (2000), pp. 3-20.

Copyright (C) by SIAM. Unauthorized reproduction of this article is prohibited. 
[28] J. G. OxLey, Matroid Theory, The Clarendon Press, Oxford University Press, New York, 1992.

[29] C. S. J. A. Nash-Williams, An application of matroids to graph theory, in Theory of Graphs (Internat. Sympos., Rome, 1966), Dunod, Paris, 1968, pp. 263-265.

[30] E. Ross, Geometric and Combinatorial Rigidity of Periodic Frameworks as Graphs on the Torus, Ph.D. thesis, York University, Toronto, Ontario, Canada, 2011.

[31] E. Ross, The rigidity of periodic body-bar frameworks on the three-dimensional fixed torus, Phil. Trans. R. Soc. A Math. Phys. Eng. Sci., 372 (2014), 20120112.

[32] S.-I. Tanigawa, Generic rigidity matroids with Dilworth truncations, SIAM J. Discrete Math., 26 (2012), pp. 1412-1439.

[33] S.-I. TANigaWA, Matroids of Gain Graphs in Applied Discrete Geometry, preprint, arXiv:1207.3601, 2012.

[34] T.-S. TAY, Rigidity of multigraphs I: Linking rigid bodies in n-space, J. Combin. Theory Ser. B, 26 (1984), pp. 95-112.

[35] T.-S. TAY, Linking $(n-2)$-dimensional panels in $n$-space II: $(n-2,2)$-frameworks and body and hinge structures, Graphs Combin., 5 (1989), pp. 245-273.

[36] T.-S. TAY, Linking $(n-2)$-dimensional panels in $n$-space I: $(k-1, k)$-graphs and $(k-1, k)$ frames, Graphs Combin., 7 (1991), pp. 289-304.

[37] M. F. Thorpe, M. V. Chubynsky, B. M. Hespenheide, S. Menor, D. J. Jacobs, L. A. Kuhn, M. I. Zavodszky, M. Lei, A. J. Rader, And W. Whiteley, Flexibility in biomolecules, in Current Topics in Physics, R. A. Barrio and K. K. Kaski, eds., Imperial College Press, London, 2005, pp. 97-112.

[38] H. Weyl, Symmetry, Princeton University Press, Princeton, NJ, 1952.

[39] W. Whiteley, The union of matroids and the rigidity of frameworks, SIAM J. Discrete Math., 1 (1988), pp. 237-255.

Copyright (c) by SIAM. Unauthorized reproduction of this article is prohibited. 